\title{
Title: The Face Mask and the Embodiment of Stigma
}

\author{
Authors: ZhiMin Xiao ${ }^{1,2, *}$, William Henley², Christopher Boyle ${ }^{1}$, Yuan Gao ${ }^{3,4}$, Justin Dillon ${ }^{1}$
}

\section{Affiliations:}

${ }^{1}$ Graduate School of Education, University of Exeter, St Luke's Campus, Exeter, EX1 2LU, UK.

${ }^{2}$ Institute of Health Research, College of Medicine and Health, University of Exeter, St Luke's Campus, Exeter, EX1 2LU, UK.

${ }^{3}$ Institute of Biomedical and Clinical Science, College of Medicine and Health, University of Exeter, St Luke's Campus, Exeter, EX1 2LU, UK.

${ }^{4}$ School of Pharmacy, Harbin University of Commerce, Harbin, Heilongjiang, 150076, China.

*Correspondence to: z.m.xiao@exeter.ac.uk.

Abstract: The COVID-19 pandemic has spawned a rare opportunity to study some latent social structures using data science. The Chinese government and its people have been blamed for the outbreak of the virus. Face mask wearing can signal an embodied stigma and Chinese people living outside China have been subject to discrimination, assault, and other hate crimes, particularly at the early stages of the crisis. However, as we accumulate more evidence surrounding mask use, the stigma is shifting. As more scientific data become available and people leave even more information on social media during the lockdown, data science can help better understand the trajectories of the stigma. The insights generated have implications for antistigma interventions for future undesirable conditions and diseases.

One Sentence Summary: Data science can help better understand the trajectories of the stigma associated with face mask wearing amongst the Chinese community living outside China.

Main Text: The COVID-19 pandemic continues to have catastrophic consequences. History shows us that after a society reluctantly acknowledges the outbreak of an epidemic, blame often follows closely. Subsequent interventions and/or the absence of essential services can be as dramatic, disruptive, or as deadly as the disease itself (1). In this pandemic, the Chinese nation and Chinese nationals were initially blamed for the outbreak, and face mask wearing amongst the Chinese community in the UK and elsewhere often signaled an embodied stigma, as it identified people as being linked to the virus (see https://bit.ly/3dx9xci for an example). As the pandemic resolves, the stigma associated with the disease and mask wearing also evolves. Studying the trajectories of the stigma is as important as the study of the virus itself, as the insights gained have implications for anti-stigma interventions for future undesirable conditions and diseases.

Stigma is a social (2) and psychological process (3). As individuals wearing face masks during COVID-19 pass from a "normal" (e.g., in Asia) to a "discredited" or "discreditable" social status (4) (e.g., in the UK), the actions and reactions surrounding mask use are embedded within macrosocial structures that shape the moral experiences of both the stigmatised and those who stigmatise. For instance, people first identify and label individuals who deviate from the norm. This stereotyping process provides fertile ground for those who wish to distance or separate "them" from "us" (5). In essence, people of a particular ethnic group are "othered" and become collectively labelled as different and thus are outside the artificial notion of being, say, British. The stigmatised group may then experience discrimination, assault, or other hate crimes. 
Stigma evolves, often as a result of targeted anti-stigma interventions and/or accumulated knowledge about a stigmatised condition, such as AIDS, smoking, and depression. To combat COVID-19, the UK government, for example, claims to base every decision on the best scientific evidence available ( 0 ), but the evidence surrounding face masks has been hotly debated (7). The UK was reluctant to recommend the public use of face masks. But the policy changed as data became increasingly available for scientific research, of particular note is the DELVE initiative of the Royal Society (https://bit.ly/2Xv7bW4). As emerging evidence suggests face covering is effective in reducing onward transmission by asymptomatic and pre-symptomatic wearers ( 8 ), one cannot help but wonder if the delay in adopting the policy in the UK is connected to institutional discrimination or prejudice, given earlier stigma associated with mask use and that many other countries acted very differently. If the connection is valid then this is a problem that might repeat in the future. To test the hypothesis and fill in the gap in evidence on mask use, we must analyse multiple types of data and utilise diverse research designs to study the social causes and consequences of the stigma and its evolving trajectories towards de-stigmatisation.

With strides made in causal machine learning, medical statistics, and digital anthropology, stigma is more researchable than before using a combination of quantitative and qualitative methods and from a multidisciplinary perspective (5). For example, data scientists can scrape the contents of social media platforms such as Twitter and Instagram to gather and analyse data on attitudes ( 9 ) towards face masks and the Chinese community since this crisis began. However, social media data will be biased, partly because it cannot sufficiently capture the true scale of discrimination - not everyone will publicly speak their mind. To solve the problem, an online experiment can be designed to collect data from non-Chinese people. The experiment should ask how many (not which) of the statements listed upset participants (see 10 for an example) using two sets of statements. The first set has some general statements about COVID-19 that would equally upset many people, for example, lack of personal protective equipment for frontline health workers, the fact that people are dying in care homes, and the number of deaths in official statistics being unable to capture the true mortality rate. The second set has one more statement saying, for example, Chinese people wear face masks everywhere. The two sets of statements should then be randomly distributed to a representative sample. The results of the experiment and the scraped data can help understand the macrosocial context and prevalence of discrimination against the Chinese community.

To examine if any prejudice detected has any scientific basis, researchers may also analyse data from different countries adopting different policies concerning face covering at different stages to understand how patterns of contracting, confirming, and recovering from the disease compare. These datasets may help data scientists identify a potential causal link if they treat face mask wearing as a natural experiment. Wearing face masks is mandatory in East Asia (11), whereas in the UK the attitude has been very different (12). However, causal inference based on observational data is challenging and mask use is only one of many response measures that have been applied. Where possible, researchers should account for measurable confounding 40 factors including the population age structure, gender, and the presence of other risk factors, such as existing health conditions, smoking, and pollution. Ideally, researchers should consider utilising data from Randomised Controlled Trials (RCTs) on the efficacy of face masks in slowing the spread of the virus (for example, the associated dataset from 13 is available). This means exploring the potential for conducting joint analysis of RCT and observational data using, for example, shrinkage methods based on heterogeneity (or agreement) weighting (14). 
Sensitivity analysis methods (15) may also be employed to assess the impact of assumptions about other control measures, including those relating to respiratory etiquette or hand hygiene.

Meanwhile, it is crucial to pay attention to COVID-19 related stories we hear and watch on a daily basis (7) and, recruit the stigmatised for in-depth interviews about their lived experiences, particularly issues surrounding face masks, any stigma they experienced and perceived, and the impacts it might have had on their wellbeing. A better understanding of face mask efficacy can help mitigate, if not eliminate, the stigma associated with mask use, just as the tide turned when science revealed the nature of human immunodeficiency virus and effective drugs became available (2). The data science approach described above is unique and valueadding in that it involves existing observational and experimental data from different countries to establish a potential causal link, which can support decision making in a timely fashion.

The impact of the research we suggest cannot be fully appreciated without an examination of the costs of its absence. Bearing the brunt that they are morally tainted when wearing face masks and a mortal threat to others, affected individuals may avoid reaching out for help, conceal their contagious status, or even stop following government advice, a response called "reverse stigmatisation" (5), for which society pays in the cost of a further spread and in the loss of more lives. When shunned and stressed, isolated individuals may be unable to shoulder the moral opprobrium and "the secondary self-imposed stigma" (2) will only get worse, particularly when their plight is compounded by the rejection of their own community. More importantly, understanding how the stigma evolves has far-reaching implications for anti-stigma interventions for future undesirable conditions and diseases.

\section{References and Notes:}

1. David S. Jones, History in a Crisis — Lessons for Covid-19. N. Engl. J. Med., 1-3 (2020).

2. G. T. Keusch, J. Wilentz, A. Kleinman, Stigma and global health: Developing a research agenda. Lancet. 367, 525-527 (2006).

3. E. Goffman, Stigma: Notes on the Management of Spoiled Identity (Simon \& Schuster, New York, 1963).

4. A. Kleinman, R. Hall-Clifford, Stigma: A social, cultural and moral process. J. Epidemiol. Community Health. 63, 418-419 (2009).

5. B. G. Link, J. C. Phelan, Stigma and its public health implications. Lancet. 367, 528-529 (2006).

6. R. Horton, Offline: COVID-19—a reckoning. Lancet. 395, 935 (2020).

7. T. Greenhalgh, Face coverings for the public: Laying straw men to rest. J. Eval. Clin. Pract., 1-8 (2020).

8. K. A. Prather, C. C. Wang, R. T. Schooley, Reducing transmission of SARS-CoV-2. Science (80-. ). 6197, 1-5 (2020).

9. C. Nobata, J. Tetreault, A. Thomas, Y. Mehdad, Y. Chang, Abusive language detection in online user content. 25th Int. World Wide Web Conf. WWW 2016, 145-153 (2016).

10. J. H. Kuklinski et al., Racial Prejudice and Attitudes Toward Affirmative Action. Am. J. Pol. Sci. 41, 402 (1997). 
11. K. K. Cheng, T. H. Lam, C. C. Leung, Wearing face masks in the community during the COVID-19 pandemic: altruism and solidarity. Lancet. 2019, 2019-2020 (2020).

12. T. Greenhalgh, M. B. Schmid, T. Czypionka, D. Bassler, L. Gruer, Face masks for the public during the covid-19 crisis. BMJ. 369, 1-4 (2020).

13. N. H. L. Leung et al., Respiratory virus shedding in exhaled breath and efficacy of face masks. Nat. Med. (2020), doi:10.1038/s41591-020-0843-2.

14. C. Röver, T. Friede, Dynamically borrowing strength from another study through shrinkage estimation. Stat. Methods Med. Res. 29, 293-308 (2020).

15. N. X. Lin, S. Logan, W. E. Henley, Bias and sensitivity analysis when estimating treatment effects from the cox model with omitted covariates. Biometrics. 69, 850-860 (2013).

Acknowledgments: Z.M.X. is supported by a University of Exeter-Alan Turing Institute research grant; Competing interests: We declare no competing interests. 